\title{
Progressive Preretinal Fibrosis with Late, Ossifying, Proliferative Retinopathy following Treatment for Retinoblastoma
}

\author{
Minh Nguyen $^{\mathrm{a}}$ Jessica Saunders $^{\mathrm{b}}$ M. Cristina Pacheco ${ }^{\mathrm{b}, \mathrm{c}}$ Andrew W. Stacey ${ }^{\mathrm{a}, \mathrm{d}}$ \\ ${ }^{a}$ Department of Ophthalmology, University of Washington, Seattle, WA, USA; ${ }^{b}$ Department of Pathology, University \\ of Washington, Seattle, WA, USA; ' Department of Laboratories, Seattle Children's Hospital, Seattle, WA, USA; \\ ${ }^{\mathrm{d}}$ Division of Ophthalmology, Seattle Children's Hospital, Seattle, WA, USA
}

\section{Established Facts}

- Intravitreal melphalan is an effective treatment modality for vitreous seeds in retinoblastoma

- Rare reported ocular side effects of intravitreal melphalan include: transient conjunctivitis, keratitis, corneal edema, increased intraocular pressure, iris atrophy, cataract, vitreous hemorrhage, preretinal hemorrhage, retinal vasculitis, chorioretinal atrophy, serous retinal detachment, and neovascularization of the optic nerve.

\section{Novel Insights}

- This paper describes progressive preretinal fibrosis starting immediately after intravitreal melphalan treatment. Clinically, the eye eventually developed acute hemorrhagic retinopathy.

- Microscopically, we found proliferative retinopathy with dystrophic ossification in the enucleated eye.

\section{Keywords}

Retinoblastoma $\cdot$ Vitreous seeding $\cdot$ Intravitreal melphalan · Preretinal fibrosis · Proliferative retinopathy

\section{Abstract \\ We report a case of retinal atrophy and progressive prereti- nal fibrosis in an eye previously treated with intravenous and intra-arterial chemotherapy (IAC), which evolved immedi- ately after treatment with intravitreal injection of melphalan. The atrophy and fibrosis progressed later to proliferative ret- inopathy with dystrophic ossification. The patient was origi-}

nally diagnosed with bilateral retinoblastoma at 4 months of age and was treated with systemic chemotherapy followed by IAC. New vitreous seeds developed and required treatment with intravitreal chemotherapy. There was resolution of vitreous seeding after 2 doses of intravitreal melphalan, but clinically the eye developed new, widespread retinal atrophy and fibrosis within 1 month of the second injection. This was followed by phthisis and late proliferative retinopathy nearly 1 year later. Retinoblastoma specialists should be aware of this potential complication of combined chemotherapy treatments.

(c) 2020 S. Karger AG, Basel

$\begin{aligned} & \text { karger@karger.com } \\ & \text { www.karger.com/oop }\end{aligned}$
Karger ${ }^{\prime /}$

Minh Nguyen

Department of Ophthalmology, University of Washington

908 Jefferson Street, 8th Floor

Seattle, WA 98104 (USA)

minhng88@uw.edu 


\section{Introduction}

In recent years, chemotherapy for the treatment of retinoblastoma has been used in various delivery routes, including intravenous, intra-arterial, and intravitreal administration, depending on the location and stage of retinoblastoma [1]. The use of intravitreal melphalan for the treatment of vitreous seeds has become increasingly em- ployed after it was established as safe and effective [2, 3]. Despite the well-established tumor control rate, knowledge of the side effect profile of intravitreal melphalan is expanding. Known ocular side effects of intravitreal injection of melphalan range from mild transient conjunctivitis to serious retinal detachment and chorioretinal atrophy [4]. Here, we report a case of clinical acute retinal atrophy, progressive preretinal fibrosis, and subsequent
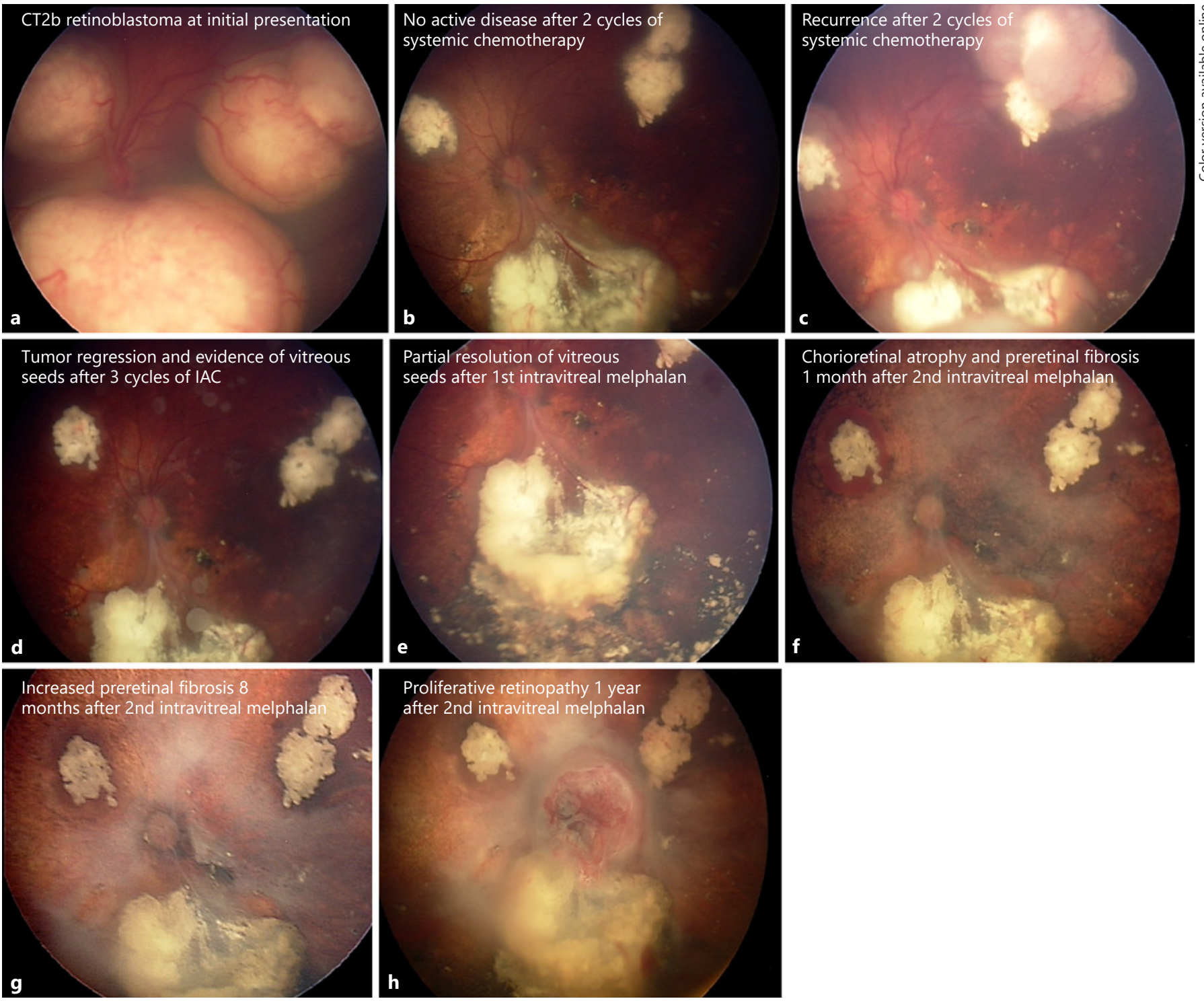

Fig. 1. Fundus photos showing the progression of retinoblastoma and complications related to the treatment of the left eye. a Initial presentation of the left eye in August 2016. b No active disease after 2 cycles of systemic chemotherapy in October 2016. c Recurrence after 5 cycles of systemic chemotherapy in February 2017. d Regression of the main tumor after 3 cycles of intra-arterial chemotherapy but new vitreous seeds found in November 2017. e Par- tial resolution of vitreous seeds after 1 dose of intravitreal melphalan as seen in January 2018. f Chorioretinal atrophy and early preretinal fibrosis found in March 2018, 1 month after the second dose of intravitreal melphalan. $\mathbf{g}$ Increased preretinal fibrosis noted in October 2018. h Proliferative retinopathy seen in January 2019, a year since the last intravitreal injection of melphalan.
Preretinal Fibrosis and Proliferative Retinopathy in Retinoblastoma Treatment
Ocul Oncol Pathol 2021;7:44-47 DOI: $10.1159 / 000509809$ 
late, histological proliferative retinopathy with dystrophic ossification following intravitreal chemotherapy injection in an eye previously treated with systemic and intra-arterial chemotherapy (IAC).

\section{Case Presentation}

A 4-month-old male child presented with bilateral retinoblastoma, cT2b, in both eyes based on the American Joint Commission on Cancer 8th edition guidelines (Fig. 1a) [5]. He was treated with 5 cycles of systemic vincristine, carboplatin, and etoposide with good initial response (Fig. 1b). Due to recurrent tumor growth (Fig. 1c), IAC with melphalan (3 mg), carboplatin (30 mg), and topotecan $(0.3 \mathrm{mg})$ was started in both eyes. He underwent a total of 3 treatments in the right eye and 4 treatments in the left eye. $\mathrm{He}$ also received laser consolidation with periodic $810-\mathrm{nm}$ diode laser and cryotherapy in both eyes. Four months after the final IAC treatment in the left eye, the patient developed multiple vitreous seeds in sphere formation (Fig. 1d). He was treated with intravitreal injection of $20 \mu \mathrm{g}$ of melphalan following established guidelines [3]. He returned 4 weeks later with significant regression, but continued to have modest residual vitreous seeding (Fig. 1e). A second intravitreal injection of $20 \mu \mathrm{g}$ melphalan was performed. At the 4-week follow-up, there was no active vitreous disease in the left eye but development of retinal atrophy and preretinal fibrosis, not previously identified, was present (Fig. 1f). Subsequent examinations revealed clinical progression of the preretinal fibrosis as well as a slow progression to phthisis (Fig. 1g). Eleven months after the second injection of intravitreal melphalan, and during a routinely scheduled exam under anesthesia without new complaints, the patient was found to have a new, hemorrhagic mass in the central macula (Fig. 1h). No additional treatment was administered. Due to poor visual prognosis and inability to rule out tumor recurrence, the family elected to proceed with enucleation of the left eye.

Histopathologic examination of the enucleated eye demonstrated effaced chorioretinal architecture with near complete loss of the normal retinal architecture around the optic nerve head (Fig. 2a). Adherent to the choroidal surface and extending into the vitreous cavity was a proliferation of small- to medium-sized vessels embedded within fibromyxoid stroma. The neovascularized tissue contained occasional residual retinal elements with marked gliosis, loss of photoreceptors, disorganization, and dissociation from the pigmented retinal epithelium in the area over and surrounding optic nerve insertion (Fig. 2b). The neovascularized tissue showed prominent calcifications and spicules of dystrophic ossification (Fig. 2c, d). Vessels were surrounded by numerous pigmented cells, some of which were hemosiderin-laden macrophages indicative of prior hemorrhage, while others were likely due to pigment migration from the disrupted retina and choroid (Fig. 2e, f). A hypercellular area is described adjacent to the optic nerve head (Fig. 2a, b), and this was felt to represent disorganized retina and retinal gliosis. Another possibility would be forced differentiation of the tumor, which has been described to occur after chemotherapy $[6,7]$. No features of proliferative residual retinoblastoma (apoptosis, mitosis, necrosis, and rosette formation) were identified in any area of the resection. The patient did well following surgery and maintains stable scars in the right eye and a normal anophthalmic socket on the left 14 months after enucleation.
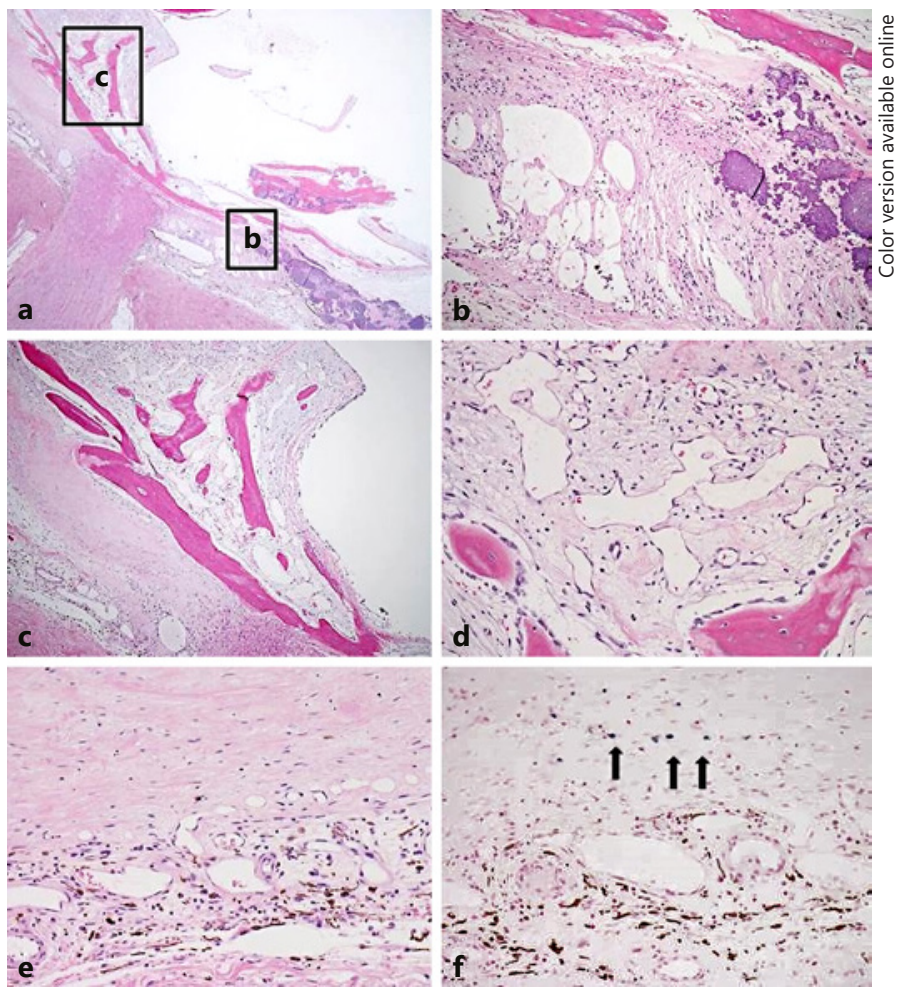

Fig. 2. Proliferative retinopathy after intravitreal melphalan injection. a Optic nerve head with area of neovascular proliferation and ossification in the upper left and calcification intermixed with residual hypercellular disorganized retinal tissue in the lower right. $\times 20$. b Calcification and ossification with disorganized and gliotic retinal tissue. $\times 100$. c. $\times 40$. d Loss of retina with dystrophic ossification and prominent neovascularization. $\times 100$. e Vascular proliferation with pigment. $\times 200$. H\&E. f Iron stain, hemosiderin-laden macrophages, blue from prior bleeding with arrows (upper half of the photo) with residual retinal pigment (brown; lower half of the photo). $\times 200$.

\section{Discussion}

As treatment options for retinoblastoma have increased, the rate of eye salvage has also increased. However, as more chemotherapy is delivered into eyes, we are learning more about the toxicity these medications can cause to the eye. Intra-arterial melphalan has been associated with tumor necrosis and calcification $[8,9]$. Pathological reports of enucleated eyes following intravitreal melphalan have shown severe gliosis, retinal necrosis, hemorrhage, and phthisis, but these were eyes treated with higher doses of melphalan $(50 \mu \mathrm{g})$ and had not received intravascular melphalan [10]. Aziz et al. [11] recently reported 2 cases of hemorrhagic retinopathy, though these occurred only 1 week after a second intravitreal melphalan injection. Sys- 
temic chemotherapy with laser consolidation has also been reported to result in glial proliferation [12]. In this case, our patient was treated with all of the above therapies: systemic chemotherapy with laser consolidation, salvage IAC, and intravitreal melphalan. The resulting toxicity was noted abruptly after the second injection of intravitreal melphalan. We expect the combination of all therapies played a role in the toxicity, though the intravitreal melphalan appears to be temporally correlated with the acute retinal atrophic and fibrotic changes. The combination of all treatments likely also played a role in the development of the late proliferative retinopathy presentation.

In our case, $20 \mu \mathrm{g}$ of melphalan was used for each intravitreal injection. Previous reports of retinal toxicities were seen after significantly higher dose $(25-50 \mu \mathrm{g})[10,11]$. The technique employed here was the same as that laid forth by Munier et al. [3] and Ghassemi and Amoli [10] and entailed the use of a 32-gauge needle with direct visualization in the vitreous space. An optical coherence tomography image was not obtained at either the first or second injection, which may have demonstrated the development of a vitreous detachment after the first injection and allow more melphalan to be injected into the subhyloid space. However, if this had been the case, one would expect a more acutely hemorrhagic effect, as seen in the 2 patients reported by Aziz et al. [11]. It is also interesting that these patients, as well as the patient presented here, all developed adverse effects after the second injection [10].

This case demonstrates that preretinal fibrosis and retinal atrophy can occur shortly after intravitreal melphalan, even at low doses $(20 \mu \mathrm{g})$. Late hemorrhage retinopa- thy may present in an eye with progressive retinal fibrotic changes nearly 1 year after any treatment of active disease. These events occurred after the eye had been treated with intravenous chemotherapy and IAC, which may play a role in the genesis of the adverse events. Retinoblastoma experts should be aware of the risk of preretinal fibrosis and its progression to hemorrhagic retinopathy in patients treated with intravitreal melphalan in combination with other chemotherapy treatments.

\section{Statement of Ethics}

The parents of the patient have given their written informed consent to publish this case report. This report also adhered to the tenets of the Declaration of Helsinki.

\section{Conflict of Interest Statement}

The authors have no financial interests to disclose.

\section{Funding Sources}

This study was supported by an unrestricted grant from Research to Prevent Blindness Inc NYC to the University of Washington Department of Ophthalmology.

\section{Author Contributions}

All authors contributed equally.

\section{References}

1 Shields C, Fulco E, Arias J, Alarcon C, Pellergrini M, Rishi P, et al. Retinoblastoma frontiers with intravenous, intra-arterial, periocular, and intravitreal chemotherapy. Eye (Lond). 2013 Feb;27(2):253-64.

2 Ghassemi F, Shields CL. Intravitreal melphalan for refractory or recurrent vitreous seeding from retinoblastoma. Arch Ophthalmol. 2012 Oct;130(10):1268-71.

3 Munier FL, Soliman S, Moulin AP, Gaillard MC, Balmer A, Beck-Popovic M. Profiling safety of intravitreal injections for retinoblastoma using an anti-reflux procedure and sterilisation of the needle track. Br J Ophthalmol. 2012 Aug;96(8):1084-7.

4 Smith SJ, Smith BD, Mohney BG. Ocular side effects following intravitreal injection therapy for retinoblastoma: a systematic review. Br J Ophthalmol. 2014 Mar;98(3): 292-7.
5 Amin M, Edge S, Greene F, Byrd D, Brookland $\mathrm{R}$, Washington $\mathrm{M}$, et al, editors. AJCC cancer staging manua. 8th ed. Springer International Publishing; 2017.

6 Bechrakis NE, Bornfeld N, Schueler A, Coupland SE, Henze G, Foerster MH. Clinicopathologic features of retinoblastoma after primary chemoreduction. Arch Ophthalmol. 1998 Jul;116(7):887-93.

7 Demirci H, Eagle RC Jr, Shields CL, Shields JA. Histopathologic findings in eyes with retinoblastoma treated only with chemoreduction. Arch Ophthalmol. 2003 Aug;121(8): 1125-31.

8 Vajzovic LM, Murray TG, Aziz-Sultan MA, Schefler AC, Fernandes CE, Wolfe SC, et al. Clinicopathologic review of enucleated eyes after intra-arterial chemotherapy with melphalan for advanced retinoblastoma. Arch Ophthalmol. 2010 Dec;128(12):1619-23.
9 Silva RA, Dubovy SR, Hess D, Stratton R, Murray $\mathrm{TG}$. Hemorrhage as a sign of treatment failure after intra-arterial chemotherapy in retinoblastoma. J AAPOS. 2015 Jun;19(3):268-70.

10 Ghassemi F, Amoli FA. Pathological findings in enucleated eyes after intravitreal melphalan injection. Int Ophthalmol. 2014 Jun;34(3): 533-40.

11 Aziz HA, Kim JW, Munier FL, Berry JL. Acute hemorrhagic retinopathy following intravitreal melphalan injection for retinoblastoma: a report of two cases and technical modification to enhance the prevention of retinal toxicity. Ocul Oncol Pathol. 2017 Jan;3(1):34-40.

12 Zolfaghari E, Kim JW, Krishnan S, ChévezBarrios P, Berry JL. Atypical retinal pigment epithelial hyperplasia and glial proliferation masquerading as progressive recurrent retinoblastoma: a case review and clinicopathologic correlation. Ocul Oncol Pathol. 2018 Sep;4(2):116-21. 